\title{
SOME NEW NONLINEAR RETARDED INTEGRAL INEQUALITIES AND THEIR APPLICATIONS
}

\author{
QING-HuA MA AND JosiP PEČARIĆ
}

\begin{abstract}
In the present paper, priori bound on certain new retarded integral inequalities in two independent variables are established. Applications are given to illustrate the usefulness of these inequalities.
\end{abstract}

Mathematics subject classification (2000): 26D15, 26D20.

Key words and phrases: retarded integral inequality, two independent variables, priori bound, partial differential equation.

\section{REFERENCES}

[1] D. Bainov, P. Simeonov, Integral Inequalities and Applications, Kluwer Academic Publishers, Dordrecht, 1992.

[2] D. S. MitRInOviĆ, J. K. PeČARIĆ AND A. M. FinK, Inequalities Involving Functions and Their Integrals and Derivatives, Dortlrecht/Boston/London, Kluwer Academic Publishers, 1991.

[3] W. S. Cheung, Q. H. MA, Nonlinear retarded integral inequalities for functions in two variables, J. Concrete Appl. Math., 2, (2004), 119-134.

[4] O. Lipovan, A retarded integral inequality and its applications, J. Math. Anal. Appl., 285, (2003), 436-443.

[5] O. LIPOVAN, A retarded Gronwall-like inequality and its applications, J. Math. Anal. Appl., 252, (2000), 389-401.

[6] Q. H. MA, E. H. YANG, Some new nonlinear delay integral inequalities, J. Math. Anal. Appl., 252, (2000), 864-878.

[7] Q. H. MA, E. H. YANG, Some new Gronwall-Bellman-Bihari type integral inequalities with delay, Period. Math. Hungar., 44, (2002), 225-238.

[8] Q. H. MA, L. DEBNATH, A more generalized Gronwal-like integral inequality with applications, Int. J. Math. Math. Sci., 33, (2003), 927-934.

[9] Q. H. MA, E. H. YANG, Some nonlinear inequalities involving improper integrals and their discrete analogues, Appl. Math. J. Chinese Univ., Ser.B, 18, (2003), 267-275.

[10] F. W. MenG, W. N. LI, On some new integral inequalities and their applications, Appl. Math. Comput., 148, (2004), 381-392.

[11] B. G. PAChPATtE, Explicit bounds on cetain integral inequalities, J. Math. Anal. Appl., 267, (2002), $48-61$.

[12] B. G. PACHPATTE, On some retarded integral inequalities and applications, JIPAM. J. Inequal. Pure Appl. Math., 3, (2002), Art. 18.

[13] B. G. PACHPATTE, Inequalities for Differential and Integral Equations, Academic Press, New York, 1998.

[14] Y. G. Sun, On retarded integral inequalities and their applications, J. Math. Anal. Appl., 301, (2005), 265-275.

[15] E. H. YANG, A new integral inequality with power nonlinear and its discrete analogue, Acta Math. Appl. Sinica, 17, (2001), 233-239. 